\title{
Relationship between ovarian cancer stem cells, epithelial mesenchymal transition and tumour recurrence
}

\author{
Monica Angelica Amaya Padilla1, Mudra Binju', Graeme Wan¹, Yohan Suryo Rahmanto ${ }^{2,3}$, Pritinder Kaur ${ }^{1}$, \\ Yu Yú ${ }^{1,4}$ \\ 'School of Pharmacy \& Biomedical Science, Curtin University, Curtin Health Innovative Research Institute, Perth, WA 6102, \\ Australia. \\ ${ }^{2}$ Sidney Kimmel Comprehensive Cancer Center, Johns Hopkins Medical Institutions, Baltimore, MD 21231, USA \\ ${ }^{3}$ Department of Pathology, Johns Hopkins Medical Institutions, Baltimore, MD 21205, USA. \\ ${ }^{4}$ Division of Obstetrics \& Gynaecology, University of Western Australia Medical School, WA 6009, Australia.
}

Correspondence to: Dr. Yu Yu, School of Pharmacy \& Biomedical Science, Curtin University, Curtin Health Innovative Research Institute, Perth, WA 6102, Australia. E-mail: yu.yu@curtin.edu.au

\begin{abstract}
How to cite this article: Amaya Padilla MA, Binju M, Wan G, Rahmanto YS, Kaur P, Yu Y. Relationship between ovarian cancer stem cells, epithelial mesenchymal transition and tumour recurrence. Cancer Drug Resist 2019;2:1127-35.

http://dx.doi.org/10.20517/cdr.2019.76
\end{abstract}

Received: 15 Sep 2019 Accepted: 29 Nov 2019 Published: 19 Dec 2019

Science Editor: Miroslav Blumenberg Copy Editor: Cai-Hong Wang Production Editor: Jing Yu

\begin{abstract}
Investigating the biological processes that occur to enable recurrence and the development of chemoresistance in ovarian cancer is critical to the research and development of improved treatment options for patients. The lethality of ovarian cancer is largely attributed to the recurrence of disease with acquired chemoresistance. Cancer stem cells are likely to be critical in ovarian cancer progression, contributing to tumour malignancy, metastasis and recurrence by persisting in the body despite treatment with anti-cancer drugs. Moreover, cancer stem cells are capable of mediating epithelial-to-mesenchymal transition traits and secrete extracellular vesicles to acquire therapy resistance and disease dissemination. These attributes merit in depth research to provide insight into the biological role of ovarian cancer stem cells in disease progression and chemotherapy response, leading to the development of improved biomarkers and innovative therapeutic approaches.
\end{abstract}

Keywords: Ovarian cancer, stem cells, chemoresistance, platinum resistance, recurrence, epithelial-to-mesenchymal transition, extracellular vesicles

\footnotetext{
(c) (1)

(C) The Author(s) 2019. Open Access This article is licensed under a Creative Commons Attribution 4.0 International License (https://creativecommons.org/licenses/by/4.0/), which permits unrestricted use, sharing, adaptation, distribution and reproduction in any medium or format, for any purpose, even commercially, as long as you give appropriate credit to the original author(s) and the source, provide a link to the Creative Commons license, and indicate if changes were made.
} 


\section{INTRODUCTION}

The cancer stem cell (CSC) model has been proposed as a mechanism in which cancer cells gain the ability to persist despite the use of cytotoxic or targeted therapeutic approaches ${ }^{[1]}$. Although it is not yet clear how widely applicable this model is for different cancer types, the theory has helped to shape understanding of advanced cancers with high relapse rate after treatment. Applying the CSC theory in clinical translation is hampered by a lack of consensus on reliable CSC markers and their dynamic behaviour.

The focus of this article is ovarian cancer-stem like cells. We hypothesise that advanced epithelial ovarian cancer (OC) is a suitable model to explore CSC theory because of the high rate of tumour recurrence after conventional treatment. High-grade serous ovarian carcinoma is a major histological subtype of epithelial OC and the leading cause of burden among gynaecological cancers. Although patients usually have a positive response to first-line treatment, the cause for concern is the recurrence of disease that $70 \%$ of patients with advanced OC experience ${ }^{[2]}$.

The standard treatment option offered to OC patients involves chemotherapy with a platinum-based agent, although most patients eventually develop resistance to it once the disease recurs. Delving deeper into the mechanisms and the biological processes that enable recurrence and resistance is vital to the development of improved treatment options. Many hypothesise that the role of CSCs is critical to the recurrence of OC and could also encourage the development of chemoresistance ${ }^{[1]}$. Previously, we outlined a number of ovarian cancer stem cells (OCSCs) markers and their role in drug resistance ${ }^{[3]}$. In this review, we expand our discussion by exploring where the CSC model comes into play in OC recurrence, the metastatic process of $\mathrm{OC}$ and its crosstalk to promote cancer progression through mechanisms involving extracellular vesicles (EVs).

\section{THE ONSET OF OVARIAN CANCER RECURRENCE AND CANCER STEM CELLS HYPOTHESIS}

OC refers to the general anatomical location from which the cancer is derived; this can include the fallopian tubes, omentum, peritoneum and other surrounding tissues in addition to the ovaries. OC is typically diagnosed at advanced stages (III or IV), at which point the cancer has affected both ovaries and/ or peritoneal metastasis has begun ${ }^{[4]}$. The dissemination of OC occurs through malignant ascites within the peritoneal cavity which lacks an anatomical barrier to obstruct the path of metastasis. The result is that solid tumour nodules spread and attach at secondary sites such as the omentum, intestines, spleen, bladder, etc. Despite being diagnosed at advanced stages of the disease, primary intervention with platinumbased therapy is initially effective in $>80 \%$ of epithelial OC patients ${ }^{[5]}$. Notably, standard treatment of OC with paclitaxel, cisplatin and carboplatin elicits a positive response in patients; however, only $10 \%-15 \%$ achieve full remission of the disease long-term ${ }^{[6]}$. It is for this reason that the lethality of OC is attributed to its recurrence with acquired chemoresistance in approximately $70 \%$ of advanced OC patients ${ }^{[2,7]}$. The recurrence of $\mathrm{OC}$ is characterised in terms of the patient's platinum-free interval (PFI). The PFI defines the period of time elapsed between the completion of platinum-based chemotherapy to the detection of tumour relapse and importantly and is an accurate reflection of the patient's anticipated response to secondline chemotherapy ${ }^{[8]}$. Subsequently, the PFI is utilized to determine the optimal second-line treatment regimen for the patient. Intervals are divided into four timeframes, namely "platinum refractory", "platinum resistant", "platinum sensitive" and "partially platinum sensitive", where relapse occurs either within four weeks, less than six months, more than six months and within 6-12 months, respectively ${ }^{[9,10]}$. Patients who have developed chemoresistance to platinum-based agents have limited options, as the routinely used chemotherapeutic approaches would be ineffective. Further research into how OC cells acquire platinum resistance is essential when it comes to opening the door to improved treatment options for these patients.

The CSC hypothesis presents the idea that a subset of tumorigenic cells has the capacity to drive tumour progression and metastasis, consequently leading to relapse in patients ${ }^{[11]}$. Abubaker et al ${ }^{[12]}$ administered in vitro chemotherapy to OVCA433 and HEY cell lines with cisplatin and paclitaxel. The results demonstrated 
Table 1. The significance of OCSCs to recurrence and chemoresistance

\begin{tabular}{|c|c|c|c|}
\hline Marker & Significance & Cell lines/Specimens & Ref. \\
\hline \multirow[t]{2}{*}{ CD24 } & $\begin{array}{l}\text { Cytoplasmic localisation of } \mathrm{CD} 24^{+} \text {is indicative of poor prognosis and } \\
\text { predictive of recurrence }\end{array}$ & Human ovarian serous tumours & [16] \\
\hline & $\begin{array}{l}\text { Increased metastasis and chemoresistance in } \mathrm{CD}_{2} 4^{+} \text {cells that have } \\
\text { developed cisplatin-resistance }\end{array}$ & CAOV-3 cell line & [17] \\
\hline CD44 & $\mathrm{CD} 44^{+} / \mathrm{CD} 24^{-}$expression in cells is predictive of recurrence & Human OC cells isolated from ascites & [18] \\
\hline \multirow[t]{2}{*}{ CD117 } & $\mathrm{CD}_{117^{+}} / \mathrm{c}-\mathrm{Kit}^{+}$expression in cells is characteristic of chemoresistance & Human advanced serous ovarian carcinoma & [19] \\
\hline & Therapy with paclitaxel increases CD177 expression in vivo & Primary tumour xenografts & [20] \\
\hline CD133 & Higher cisplatin $\mathrm{IC}_{50}$ in $\mathrm{CD}_{133^{+}}$cells characteristic of chemoresistance & 42 established OC cell lines & [21] \\
\hline ALDH & $\begin{array}{l}\text { ALDH1A1 expression in OCSCs correlates with poor survival and } \\
\text { chemoresistance }\end{array}$ & Human high-grade OC tumours & [13] \\
\hline
\end{tabular}

OC: ovarian cancer; OCSCs: ovarian cancer stem cells

an enhanced expression of CSC markers at both protein and mRNA levels post-chemotherapy ${ }^{[12]}$. Additionally, other researchers have corroborated the increased expression of CSC markers postchemotherapy, reinforcing this finding ${ }^{[12-14]}$. In terms of relapse, it is important to evaluate the impact of CSCs because even patients who demonstrate a positive response to chemotherapy could relapse if a small population of CSCs were to persist ${ }^{[15]}$. Research has linked the presence of CSCs to chemoresistance and recurrence in OC based on the expression of stem cell markers such as CD24, CD44, CD117, $\mathrm{CD} 133$ and aldehyde dehydrogenase $(\mathrm{ALDH})^{[3,13,16-21]}$. These findings reinforce the importance of CSC markers as biomarkers for the progression of OC [Table 1]. Collectively, these data suggest a relationship between the administration of platinum-based agents and the accumulation of stem cell characteristics, which in turn promote recurrence. However, further research must be done to better understand the efficacy of treatment and to ascertain the prognostic significance of using these markers. It is vital to evaluate the molecular mechanisms surrounding CSCs and their association with OC recurrence.

\section{EPITHELIAL-MESENCHYMAL TRANSITION OF OVARIAN CANCER CELLS}

Epithelial-to-mesenchymal transition (EMT) is a biological process in which epithelial cells have diminished cell-cell adhesion and cell polarity while they acquire invasive characteristics that promote a mesenchymal phenotype ${ }^{[22]}$. In embryogenesis, organ development and tissue regeneration EMT is an essential series of events that must occur in order to achieve normal development ${ }^{[22,23]}$. In terms of cancer, EMT is highly problematic as its acquisition enables cancer cell invasion and encouragement of metastasis ${ }^{[24,25]}$. Mesenchymal and epithelial characteristics exist on a spectrum whereby cells can present intermediate traits between the two extremes, rather than being completely epithelial- or mesenchymal-like cells $^{[26]}$. The plasticity along the epithelial-mesenchymal axis permits the reverse process, which is termed mesenchymal-to-epithelial transition, shown in Figure $1^{[27]}$.

It is hypothesised that EMT is critical for OC progression. Immunohistochemical studies examining EMTrelated proteins (E-cadherin and SNAI1) in primary and metastatic ovarian carcinoma showed that the EMT status, as marked by both a reduced E-cadherin expression and positive nuclear expression of SNAI1, was significantly higher in patients with peritoneal metastasis than those without. Notably, EMT-positive status was also a predictor of poor progression free survival and overall survival ${ }^{[28]}$. In addition, other EMT related proteins, such as vimentin and ZEB1, were identified as markers of poor chemotherapy response in metastatic serous ovarian carcinoma effusions ${ }^{[2]}$. Furthermore, a number of studies suggested that OC cells resistant to carboplatin and/or paclitaxel frequently acquire mesenchymal traits ${ }^{[30-32]}$. However, the relationship between OCSCs and acquisition of the mesenchymal phenotype is poorly understood.

\section{THE IDEA OF MIGRATING CANCER STEM CELLS}

The existence of stationary and migrating CSCs was initially suggested by Brabletz et al ${ }^{[33]}$ to explain the behaviour of colon cancer. It is based on the observation that colon carcinoma gene expression can 




Figure 1. Epithelial-to-mesenchymal transition (EMT) process in cells. Diagrammatic summary depicting the effects of EMT in cells. The progression of EMT shows the upregulation of mesenchymal markers such as TWIST1, SNAI1, TGF- $\beta$, aldehyde dehydrogenase (ALDH), $\mathrm{N}$-cadherin, vimentin and other cancer stem cell (CSC) markers. Profiles with increased expression of these markers have been shown to shift towards a mesenchymal phenotype depicted as irregular shaped cells with a lack of tight junctions. mesenchymal-to-epithelial transition is the reversion of this process. Cells that have shifted toward a mesenchymal state through the upregulation of these markers often acquire stem-like features and chemoresistance

be largely grouped into a dynamic two-phase expression pattern: (1) stemness/proliferative group that is activated early and throughout the cancer progression; and (2) a EMT/dissemination group that is transiently activated later at the tumour-host interface ${ }^{[33]}$. Therefore, stationary CSCs are believed to be embedded in epithelial tissue, while mobile CSCs are predominantly at the tumour periphery at the host interface where they may readily undergo EMT to invade and metastasise, possibly through activation by environmental signals. The link between stemness and EMT is further supported by the findings that EMT activators such as TWIST1 can actually induce both EMT and "stemness" properties ${ }^{[23]}$.

Delving further into the associations between the existence of CSCs and EMT in OC, we find increasing evidence that supports the notion that OC cells with stem cell properties and/or EMT are inclined to develop resistance to therapy ${ }^{[14]}$. Moreover, the co-expression of "stemness" genes and EMT genes have been reported. Investigation of advanced-stage ovarian tumour sections, metastatic cells isolated from ascites and OC cell line revealed the combined expression of EMT and CSC markers after cisplatin treatment ${ }^{[14]}$. Cisplatin induced EMT morphology was characterised by reduced E-cadherin, as well as increased $\mathrm{N}$-cadherin and vimentin expression. At the same time, CSC markers including CD44, CD117, CD 133 and stem cell transcription factors Nanog and Oct- 4 were also expressed. These studies utilised $\alpha 2$ integrin subunit and EpCAM to further characterise the cells on a molecular level ${ }^{[14]}$. A study by Lupia et al. ${ }^{[34]}$ using primary OC cells demonstrated the co-regulation of CD73, which was enriched in OC-initiating cells with EMT-associated factors such as SNAI1, TWIST1 and ZEB1 to promote a mesenchymal-like phenotype. CD73 causes dephosphorylation of adenosine monophosphate (AMP) to adenosine triggering an increase in intracellular cyclic AMP which is suppressive of immune functionality, consequently encouraging cancer progression, metastasis and angiogenesis ${ }^{[35]}$. Thus, CD73 promotes OC stemness and $\mathrm{EMT}^{[34]}$.

Research into the heterogeneity of OC cell lines opened the discussion about OCSCs establishing heterogeneity through EMT, which could result in metastasis, tumour progression or recurrence and drug resistance. Side population (SP) cells have tumour initiating capacity and express stem-like genes with characteristics that promote drug resistance ${ }^{[36]}$. Higher levels of SP cells have been detected in OC patients' ascitic fluid, and these cells express mesenchymal gene expression patterns. The presence of these cells has been correlated with tumour grade which proves useful in a prognostic setting ${ }^{[37]}$. These observations implicate the EMT process in contributing to heterogeneity. Inhibiting the EMT process through SNAI1 
silencing reduced SP cells frequency, suggesting that SNAI1-regulated EMT may be required to maintain $\operatorname{OCSCs}^{[36]}$.

The processes of glycolysis and lipolysis are pivotal to the supply of energy to $\operatorname{CSCs}^{[38]}$. There is limited research into the relationship between EMT and these processes, however, we have highlighted some findings of interest in this review. The inhibition of glycolysis in cancer stem-like cells enriched from the SKOV3 cell line (i.e., treatment with Lonidamine) impeded the expression of EMT-associated genes (ZEB, SNAIL and vimentin $)^{[39]}$. In contrast, only moderate effect was observed when perturbing lipolysis. Thus, inhibiting glycolysis under the right conditions could be used as a CSC target to prevent EMT.

A study by Marchini and colleagues compared the molecular profiles of stage III-IV epithelial ovarian tumours before and after several lines of platinum chemotherapy. They identified resistant gene expression signatures indicative of EMT activation via the TGF $\beta$ pathway ${ }^{[38]}$. Importantly, this gene signature also negatively affected survival in OC patients ${ }^{[38]}$. In cell line studies, the exposure of OC cells to prolonged treatment with TGF $\beta 1(48 \mathrm{~h})$ resulted in the acquisition of stem-like characteristics and chemoresistant properties. TGF $\beta 1$ induced the mRNA transcription of pluripotent markers such as SOX2, OCT4a, NANOG, CD44 and CD $117^{[40]}$. Furthermore, TGF $\beta 1$ treatment can upregulate the expression of $A B C G 2$, a member of the $\mathrm{ABC}$ transporter family, at both the mRNA and protein level consistent with previous findings ${ }^{[41]}$. Upregulation of drug transporter proteins from the ABC family can be a hallmark of acquired chemoresistance given their well-documented drug efflux functions ${ }^{[42]}$. A recent report using cytotoxicity as a readout showed that human ovarian teratocarcinoma PA1 cells were susceptible to cisplatin induced cell necrosis - but not when treated with TGF $\beta 1$. This suggests that the TGF $\beta$ pathway ultimately affects the chemosensitivity of OC cells ${ }^{[41]}$. Interestingly, the use of a calcium channel blocker such as verapamil can re-sensitise TGF $\beta 1$-treated cells to the cytotoxic effects of cisplatin ${ }^{[41]}$. Analyses of the SP fraction of the HO-8910PM human epithelial OV cell line following TGF $\beta 1$ treatment revealed transformation to mesenchymal features, stimulating expression levels of the EMT-related genes SNAI1, SNAI2 and $\mathrm{CDH} 2{ }^{[43]}$. These data propose the concept of OCSC evolution via EMT, which could consequently affect chemoresistance and disease progression.

OCSCs often demonstrate high expression of NANOG which influences EMT processing ${ }^{[44]}$. NANOG utilises the STAT3 pathway to encourage EMT and drug resistance, hence increasing its expression within chemoresistant OC cell lines ${ }^{[45,46]}$. It is even postulated that NANOG expression may be an explanation for the aggressive phenotype associated with $\mathrm{OCSCs}^{[46]}$. A putative drug resistant tumour cell phenotype, $\mathrm{EpCAM}^{+} \mathrm{CD} 45^{+}$, was isolated from the ascitic fluid of OC patients. When compared to only $\mathrm{EpCAM}^{+}$ cells, the drug resistant phenotype demonstrates an increase in E-cadherin, $\mathrm{N}$-cadherin and vimentin gene expression, which suggest the promotion of EMT and an association with chemoresistance ${ }^{[4]}$. $\mathrm{EpCAM}^{+} \mathrm{CD} 45^{+}$cells have a highly invasive phenotype with mesenchymal gene signatures largely attributed to an OCSC subpopulation. This further points to the impact of EMT in CSCs and how that causes drug resistance.

ALDH is another OCSC marker where expression is linked to poor prognoses and chemoresistance in OC patients ${ }^{[13]}$. ALDH ${ }^{\text {high }}$ OC populations demonstrate CSC-like invasive properties and express CSC markers in vivo while simultaneously demonstrating advanced stages of EMT processing ${ }^{[48]}$. Collectively, these findings support and give an insight into the complex interplay among EMT, CSCs and the onset of OC chemoresistance. The underlying hypothesis behind this is that, during therapy, OC tumour cells can acquire features that are characteristic of EMT and CSC signatures that assist and promote tumour recurrence [Figure 1]. 


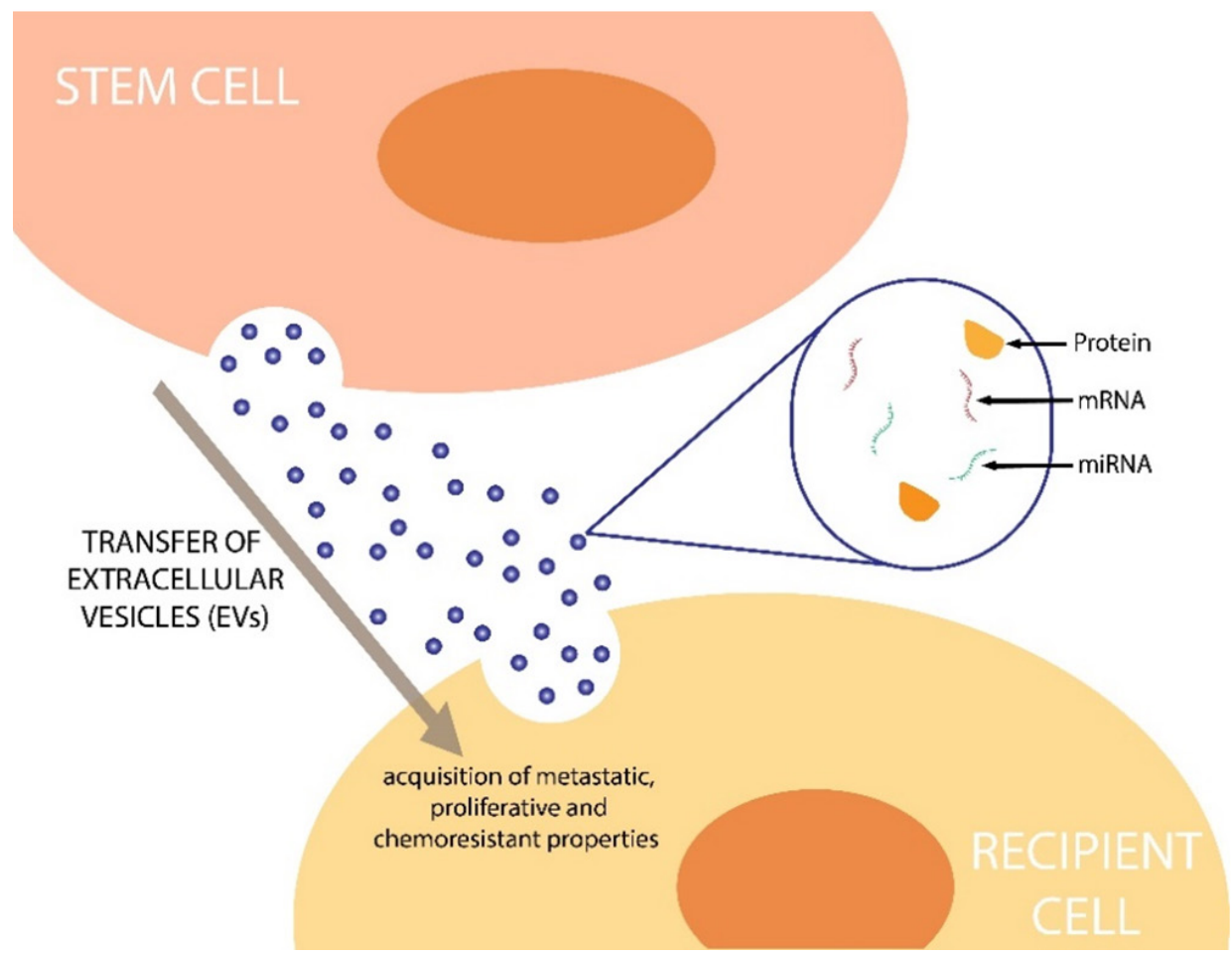

Figure 2. Transfer of extracellular vesicles (EVs) to recipient cells. A proposed model of the process that stem cells use to transfer EVs containing biological materials such as mRNAs, miRNAs and proteins, which can ultimately confer chemoresistant and cancer stem cell properties to recipient cells

\section{EXTRACELLULAR VESICLES}

Interest in EVs and their role in intercellular communication in a cancer setting has gained traction in recent years. EVs can promote tumour cell growth, proliferation and metastasis in cancer, which has made them an attractive prospect in research ${ }^{[4,50]} . \mathrm{EV}$ is a blanket term that is used to describe any secreted vesicle including microvesicles, oncosomes, exosomes and many more. EVs are secreted by many cell types and can be detected in blood and urine. The contents of EVs vary depending on the cell that secretes them. They can contain proteins, nucleic acids and other biological material ${ }^{[4]}$. The biological material enclosed in the EV is transported in the bloodstream allowing cell-cell communication via these secreted vesicles.

There have been developments in the research of cancer-associated EVs that suggest they assist the transmission of mRNAs, miRNAs and proteins, conferring chemoresistance from cell to cell in many cancers including OC [Figure 2]. An early study by Safaei and colleagues utilised the lysosomal compartment in cisplatin-sensitive 2008 human ovarian carcinoma cells to demonstrate that EVs confer chemoresistance in a cisplatin-resistant subvariant cell line ${ }^{[51]}$. In this study, the $2008 / \mathrm{C}_{1} 3^{\star} 5.25$ cisplatinresistant variant exported 2.6 -fold more cisplatin than the drug sensitive 2008 human ovarian carcinoma parental cell line ${ }^{[51]}$. When A2780 cells were treated with exosomes from the 2008/C13*5.25 cell line, they produced gene signatures coinciding with those of EMT. Crow and colleagues suggested that this may be achieved through the upregulation of EMT markers via secreted exosomes and EVs as a whole ${ }^{[52]}$.

Pluripotent stem cells have been shown to secrete EVs. For example, mouse cardiac fibroblast-derived induced pluripotent stem cells were reported to secrete cardioprotective miRNAs including NANOGregulated miR-21 and HIF- $1 \alpha$-regulated miR-210 to prevent cardiomyocyte apoptosis ${ }^{[53]}$. Similarly, CSCs may exploit EVs to promote the progression of cancer by affecting cell-cell communication ${ }^{[54]}$. Cha and colleagues $^{[55]}$ investigated miRNA dysregulation in CSCs isolated from human ovarian carcinoma tissues. 
CSCs derived from spheroid-forming primary cancer cells showed dysregulation of five miRNAs (miR-5703, miR-630, miR-1246, miR-424-5p and miR-320b) when compared to primary cancer cell expression profiles. In particular, miR-424-5p was linked to distant metastasis, suggesting a role in dissemination of disease ${ }^{[55]}$. Given that miRNAs control CSC self-renewal and differentiation, these findings suggest that miR-424-5p is a potential biomarker for poor prognosis in OC, making this an attractive target in terms of therapeutics.

A recent study by Srivastava et al ${ }^{[56]}$ suggested that miRNAs are important in maintaining CSC properties. Specifically, OCSCs exhibited upregulated miR-328-3p (miR-328) through targeting DNA damage binding protein 2. Enhanced expression of miR-328 was related to reduced ERK signalling activity in OCSC, and miR-328 inhibition prevented tumour metastasis and growth in murine ovarian xenografts ${ }^{[56]}$. These findings suggest the possibility of targeting miR-328 to eradicate CSC in OC. In cell line studies, decreased expression of miR-34c-5p in SKOV-I6 and OVS1 cells resulted in docetaxel and carboplatin drug resistance $^{[57]}$. Conversely, chemoresistant cell lines SKOV3/DDP and SKOV3 showed increased expression of miRNA-30a-5p compared to chemosensitive cell lines, reinforcing their role in chemoresistance ${ }^{[58]}$. These data demonstrate how regulatory miRNAs can be exploited to confer resistance and promote the progression of disease.

\section{CONCLUSION}

Studies have demonstrated the enrichment of CSC markers following treatment with platinum-based agents. Treatment with platinum-based agents encourages the development of CSC characteristics which promote tumour progression and metastasis, ultimately resulting in wide-spread disease recurrence unamenable to secondary surgery. We hypothesise that treatment with chemotherapeutic agents can lead to alterations in molecular signatures typical of CSCs. As outlined, there is ample evidence that supports a relationship among chemotherapy, CSCs and resistance. The issue, however, is being able to determine the extent to which tumours recur because of chemotherapy, which induces CSCs to dominate, or due to CSCs' intrinsic resistance to chemotherapy in OC. Migrating CSCs have the capacity to undergo EMT processing, negatively impacting patient disease progression. Furthermore, CSCs may promote cancer progression through stem cell associated EVs. At the same time, these cells are maintained by an EV-based mechanism that is crucial in governing cell-cell communication. It is vital that future studies investigate the interplay between CSCs and EMT in greater depth in order to understand metastatic tumour recurrence in advanced OCs.

\section{DECLARATIONS}

\section{Authors' contributions}

Reviewed literature and wrote first draft: Amaya Padilla M

Designed Figures: Amaya Padilla M

Reviewed, edited, and revised manuscript: Amaya Padilla M, Binju M, Wan G, Rahmanto YS, Kaur P, Yu Y

\section{Availability of data and materials}

Not applicable.

\section{Financial support and sponsorship}

This research was funded by Raine Priming Grant, RPG101-18, Australia (Yu Y).

\section{Conflicts of interest}

All authors declare that there are no conflicts of interest.

\section{Ethical approval and consent to participate}

Not applicable. 


\section{Consent for publication}

Not applicable.

\section{Copyright}

(c) The Author(s) 2019.

\section{REFERENCES}

1. Albini A, Bruno A, Gallo C, Pajardi G, Noonan DM, et al. Cancer stem cells and the tumor microenvironment: interplay in tumor heterogeneity. Connect Tissue Res 2015;56:414-25.

2. Ushijima K. Treatment for recurrent ovarian cancer-at first relapse. J Oncol 2010;2010:497429.

3. Pieterse Z, Amaya Padilla MA, Singomat T, Binju M, Madjid BD, et al. Ovarian cancer stem cells and their role in drug resistance. Int J Biochem Cell Biol 2019;106:117-26.

4. Berek JS, Crum C, Friedlander M. Cancer of the ovary, fallopian tube, and peritoneum. Int J Gynecol Obstet 2015;131:S111-22.

5. Kim A, Ueda Y, Naka T, Enomoto T. Therapeutic strategies in epithelial ovarian cancer. J Exp Clin Cancer Res 2012;31:14.

6. Yu Y, Gaillard S, Phillip Jude M, Huang TC, Pinto Sneha M, et al. Inhibition of spleen tyrosine kinase potentiates paclitaxel-induced cytotoxicity in ovarian cancer cells by stabilizing microtubules. Cancer Cell 2015;28:82-96.

7. Sebastian V, Jermaine IC, Robert CB, Andy B, Jonathan SB, et al. Rethinking ovarian cancer: recommendations for improving outcomes. NatRev Cancer 2011;11:719.

8. Markman M, Rothman R, Hakes T, Reichman B, Hoskins W, et al. Second-line platinum therapy in patients with ovarian cancer previously treated with cisplatin. J Clin Oncol 1991;9:389-93.

9. Luvero D, Milani A, Ledermann JA. Treatment options in recurrent ovarian cancer: latest evidence and clinical potential. Ther Adv Med Oncol 2014;6:229-39.

10. Stuart GCE, Kitchener H, Bacon M, duBois A, Friedlander M, et al. 2010 Gynecologic Cancer InterGroup (GCIG) consensus statement on clinical trials in ovarian cancer: report from the Fourth Ovarian Cancer Consensus Conference. Int J Gynecol Cancer 2011;21:750-5.

11. Rahman M, Azari H, Deleyrolle L, Vedam-Mai V, Reynolds BA, et al. The cancer stem cell hypothesis: failures and pitfalls. Neurosurgery 2011;68:531-45; discussion 545.

12. Abubaker K, Latifi A, Luwor R, Nazaretian S, Zhu H, et al. Short-term single treatment of chemotherapy results in the enrichment of ovarian cancer stem cell-like cells leading to an increased tumor burden. Molecular Cancer 2013;12:24.

13. Landen CN, Goodman B, Katre AA, Steg AD, Nick AM, et al. Targeting aldehyde dehydrogenase cancer stem cells in ovarian cancer. Mol Cancer Ther 2010;9:3186-99.

14. Latifi A, Abubaker K, Castrechini N, Ward AC, Liongue C, et al. Cisplatin treatment of primary and metastatic epithelial ovarian carcinomas generates residual cells with mesenchymal stem cell-like profile. J Cell Biochem 2011;112:2850-64.

15. Tan BT, Park CY, Ailles LE, Weissman IL. The cancer stem cell hypothesis: a work in progress. Lab Invest 2006;86:1203-7.

16. Choi YL, Kim SH, Shin YK, Hong YC, Lee SJ, et al. Cytoplasmic CD24 expression in advanced ovarian serous borderline tumors. Gynecol Oncol 2005;97:379-86.

17. Nakamura K, Terai Y, Tanabe A, Ono YJ, Hayashi M, et al. CD24 expression is a marker for predicting clinical outcome and regulates the epithelial-mesenchymal transition in ovarian cancer via both the Akt and ERK pathways. Oncol Rep 2017;37:3189-200.

18. Meng E, Long B, Sullivan P, McClellan S, Finan MA, et al. CD44+/CD24- ovarian cancer cells demonstrate cancer stem cell properties and correlate to survival. Clin Exp Metastasis 2012;29:939-48.

19. Taddei A, Villanucci A, Amunni G, Baroni G, Taddei GL, et al. c-KIT expression and correlation with chemotherapy resistance in ovarian carcinoma: an immunocytochemical study. Ann Oncol 2004;15:594-7.

20. Abubaker K, Luwor RB, Escalona R, McNally O, Quinn MA, et al. Targeted disruption of the JAK2/STAT3 pathway in combination with systemic administration of paclitaxel inhibits the priming of ovarian cancer stem cells leading to a reduced tumor burden. Front Oncol 2014;4:75.

21. Baba T, Convery PA, Matsumura N, Whitaker RS, Kondoh E, et al. Epigenetic regulation of CD133 and tumorigenicity of CD133+ ovarian cancer cells. Oncogene 2008;28:209.

22. Kalluri R, Weinberg RA. The basics of epithelial-mesenchymal transition. J Clin Invest 2009;119:1420-8.

23. Mani SA, Guo W, Liao MJ, Eaton EN, Ayyanan A, et al. The Epithelial-mesenchymal transition generates cells with properties of stem cells. Cell 2008;133:704-15.

24. Lamouille S, Xu J, Derynck R. Molecular mechanisms of epithelial-mesenchymal transition. Nat Rev Molecular Cell Biol 2014;15:178-96.

25. Thiery JP, Acloque H, Huang RYJ, Nieto MA. Epithelial-mesenchymal transitions in development and disease. Cell 2009;139:871-90.

26. Acloque H, Adams MS, Fishwick K, Bronner-Fraser M, Nieto MA. Epithelial-mesenchymal transitions: the importance of changing cell state in development and disease. J Clin Invest 2009;119:1438-49.

27. Chaffer CL, San Juan BP, Lim E, Weinberg RA. EMT, cell plasticity and metastasis. Cancer Metastasis Rev 2016;35:645-54.

28. Takai M, Terai Y, Kawaguchi H, Ashihara K, Fujiwara S, et al. The EMT (epithelial-mesenchymal-transition)-related protein expression indicates the metastatic status and prognosis in patients with ovarian cancer. J Ovarian Res 2014;7:76.

29. Davidson B, Holth A, Hellesylt E, Tan TZ, Huang RY, et al. The clinical role of epithelial-mesenchymal transition and stem cell markers in advanced-stage ovarian serous carcinoma effusions. Human Pathology 2015;46:1-8. 
30. Zhu X, Shen H, Yin X, Long L, Xie C, et al. miR-186 regulation of Twist1 and ovarian cancer sensitivity to cisplatin. Oncogene 2016;35:323-32.

31. Haslehurst AM, Koti M, Dharsee M, Nuin P, Evans K, et al. EMT transcription factors snail and slug directly contribute to cisplatin resistance in ovarian cancer. BMC Cancer 2012;12:91.

32. Chowanadisai W, Messerli SM, Miller DH, Medina JE, Hamilton JW, et al. Cisplatin resistant spheroids model clinically relevant survival mechanisms in ovarian tumors. PLoS One 2016;11:e151089.

33. Brabletz T, Jung A, Spaderna S, Hlubek F, Kirchner T. Opinion: migrating cancer stem cells - an integrated concept of malignant tumour progression. Nat Rev Cancer 2005;5:744-9.

34. Lupia M, Angiolini F, Bertalot G, Freddi S, Sachsenmeier KF, et al. CD73 regulates stemness and epithelial-mesenchymal transition in ovarian cancer-initiating cells. Stem Cell Reports 2018;10:1412-25.

35. Allard D, Allard B, Gaudreau PO, Chrobak P, Stagg J. CD73-adenosine: a next-generation target in immuno-oncology. Immunotherapy 2016;8:145-63.

36. Wu C, Alman BA. Side population cells in human cancers. Cancer Lett 2008;268:1-9.

37. Wu C, Wei Q, Utomo V, Nadesan P, Whetstone H, et al. Side population cells isolated from mesenchymal neoplasms have tumor initiating potential. Cancer Res 2007;67:8216-22.

38. Marchini S, Fruscio R, Clivio L, Beltrame L, Porcu L, et al. Resistance to platinum-based chemotherapy is associated with epithelial to mesenchymal transition in epithelial ovarian cancer. Eur J Cancer 2013;49:520-30.

39. Pouyafar A, Heydarabad MZ, Abdolalizadeh J, Rahbarghazi R, Talebi M. Modulation of lipolysis and glycolysis pathways in cancer stem cells changed multipotentiality and differentiation capacity toward endothelial lineage. Cell Biosci 2019;9:30.

40. Liu A, Yu X, Liu S. Pluripotency transcription factors and cancer stem cells: small genes make a big difference. Chinese journal of cancer 2013;32:483-7.

41. Mitra T, Prasad P, Mukherjee P, Chaudhuri SR, Chatterji U, et al. Stemness and chemoresistance are imparted to the OC cells through TGF $\beta 1$ driven EMT. J Cell Biochem 2018;119:5775-87.

42. Fletcher JI, Haber M, Henderson MJ, Norris MD. ABC transporters in cancer: more than just drug efflux pumps. Nat Rev Cancer 2010;10:147.

43. Jiang H, Lin X, Liu Y, Gong W, Ma X, et al. Transformation of epithelial ovarian cancer stemlike cells into mesenchymal lineage via EMT results in cellular heterogeneity and supports tumor engraftment. Molecular medicine (Cambridge, Mass) 2012;18:1197-208.

44. Parida S, Chakraborty S, Maji RK, Ghosh Z. Elucidating the gene regulatory networks modulating cancer stem cells and non-stem cancer cells in high grade serous ovarian cancer. Genomics 2019;111:103-13.

45. Liu S, Sun J, Cai B, Xi X, Yang L, et al. NANOG regulates epithelial-mesenchymal transition and chemoresistance through activation of the STAT3 pathway in epithelial ovarian cancer. Tumor Biology 2016;37:9671-80.

46. Qin S, Li Y, Cao X, Du J, Huang X. NANOG regulates epithelial-mesenchymal transition and chemoresistance in ovarian cancer. Biosci Rep 2017;37:BSR20160247.

47. Akhter MZ, Sharawat SK, Kumar V, Kochat V, Equbal Z, et al. Aggressive serous epithelial ovarian cancer is potentially propagated by EpCAM(+)CD45(+) phenotype. Oncogene 2018;37:2089-103.

48. Li Y, Chen T, Zhu J, Zhang H, Jiang H, et al. High ALDH activity defines ovarian cancer stem-like cells with enhanced invasiveness and EMT progress which are responsible for tumor invasion. Biochem Biophys Res Commun 2018;495:1081-8.

49. Sun YZ, Ruan JS, Jiang ZS, Wang L, Wang SM. Extracellular vesicles: a new perspective in tumor therapy. BioMed Res Int 2018;2018:2687954.

50. Alharbi M, Zuñiga F, Elfeky O, Guanzon D, Lai A, et al. The potential role of miRNAs and exosomes in chemotherapy in ovarian cancer. 2018;25:R663.

51. Safaei R, Larson BJ, Cheng TC, Gibson MA, Otani S, et al. Abnormal lysosomal trafficking and enhanced exosomal export of cisplatin in drug-resistant human ovarian carcinoma cells. Mol Cancer Ther 2005;4:1595-604.

52. Crow J, Atay S, Banskota S, Artale B, Schmitt S, et al. Exosomes as mediators of platinum resistance in ovarian cancer. Oncotarget 2017;8:11917-36.

53. Wang Y, Zhang L, Li Y, Chen L, Wang X, et al. Exosomes/microvesicles from induced pluripotent stem cells deliver cardioprotective miRNAs and prevent cardiomyocyte apoptosis in the ischemic myocardium. Int J Cardiol 2015;192:61-9.

54. Liao TT, Yang MH. Revisiting epithelial-mesenchymal transition in cancer metastasis: the connection between epithelial plasticity and stemness. Mol Oncol 2017;11:792-804.

55. Cha SY, Choi YH, Hwang S, Jeong JY, An HJ. Clinical impact of microRNAs associated with cancer stem cells as a prognostic factor in ovarian carcinoma. J Cancer 2017;8:3538-47.

56. Srivastava AK, Banerjee A, Cui T, Han C, Cai S, et al. Inhibition of miR-328-3p impairs cancer stem cell function and prevents metastasis in ovarian cancer. Cancer Res 2019;79:2314-26.

57. Tung SL, Huang WC, Hsu FC, Yang ZP, Jang TH, et al. miRNA-34c-5p inhibits amphiregulin-induced ovarian cancer stemness and drug resistance via downregulation of the AREG-EGFR-ERK pathway. Oncogenesis 2017;6:e326.

58. Liu J, Wu X, Liu H, Liang Y, Gao X, et al. Expression of microRNA-30a-5p in drug-resistant and drug-sensitive ovarian cancer cell lines. Oncol Lett 2016;12:2065-70. 